\title{
Correction to: Pin 1 inhibition exerts potent activity against acute myeloid leukemia through blocking multiple cancer-driving pathways
}

Xiaolan Lian ${ }^{1,2,3+}$, Yu-Min Lin² ${ }^{2 \dagger}$, Shingo Kozono ${ }^{2}$, Megan K. Herbert ${ }^{2}$, Xin Li ${ }^{1}$, Xiaohong Yuan ${ }^{1}$, Jiangrui Guo ${ }^{1}$, Yafei Guo ${ }^{1}$, Min Tang ${ }^{1}$, Jia Lin ${ }^{1}$, Yiping Huang ${ }^{1}$, Bixin Wang ${ }^{1}$, Chenxi Qiu' ${ }^{2}$, Cheng-Yu Tsai ${ }^{2}$, Jane Xie ${ }^{2}$, Ziang Jeff $\mathrm{GaO}^{2}$, Yong Wu', Hekun $\mathrm{Liu}^{3}$, Xiao Zhen Zhou ${ }^{2,3^{*}}$, Kun Ping $\mathrm{Lu}^{2,3^{*}}$ and Yuanzhong Chen ${ }^{{ }^{*}}$

\section{Correction}

The original article [1] contained minor typesetting errors affecting the following authors: Ziang Jeff Gao, Xiao Zhen Zhou, and Kun Ping Lu. The article has now been corrected to display the authors' names correctly.

\begin{abstract}
Author details
${ }^{1}$ Fujian Institute of Hematology, Fujian Provincial Key Laboratory on Hematology, Fujian Medical University Union Hospital, Fuzhou, China. ${ }^{2}$ Division of Translational Therapeutics, Department of Medicine and Cancer Research Institute, Beth Israel Deaconess Medical Center, Harvard Medical School, Boston, USA. ${ }^{3}$ Fujian Key Laboratory for Translational Research in Cancer and Neurodegenerative Diseases, Institute for Translational Medicine, Fujian Medical University, Fuzhou, China.
\end{abstract}

Received: 25 June 2018 Accepted: 25 June 2018

Published online: 11 July 2018

\section{Reference}

1. Lian $\mathrm{X}$, et al. Pin1 inhibition exerts potent activity against acute myeloid leukemia through blocking multiple cancer-driving pathways. J Hematol Oncol. 2018;11:73.

\footnotetext{
* Correspondence: xzhou@bidmc.harvard.edu; klu@bidmc.harvard.edu; chenyz@mail.fjmu.edu.cn

${ }^{\dagger}$ Xiaolan Lian and Yu-Min Lin contributed equally to this work.

${ }^{2}$ Division of Translational Therapeutics, Department of Medicine and Cancer

Research Institute, Beth Israel Deaconess Medical Center, Harvard Medical

School, Boston, USA

${ }^{1}$ Fujian Institute of Hematology, Fujian Provincial Key Laboratory on

Hematology, Fujian Medical University Union Hospital, Fuzhou, China

Full list of author information is available at the end of the article
}

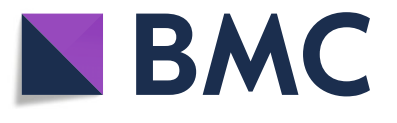

(c) The Author(s). 2018 Open Access This article is distributed under the terms of the Creative Commons Attribution 4.0 International License (http://creativecommons.org/licenses/by/4.0/), which permits unrestricted use, distribution, and reproduction in any medium, provided you give appropriate credit to the original author(s) and the source, provide a link to the Creative Commons license, and indicate if changes were made. The Creative Commons Public Domain Dedication waiver (http://creativecommons.org/publicdomain/zero/1.0/) applies to the data made available in this article, unless otherwise stated. 